\title{
Research of Situation and Countermeasures Base on net worker of Teachers and Students Relationship in University
}

\author{
Gui-ping Yuan \\ School of Education, Linyi University, Linyi(276005), Shandong Province China
}

\begin{abstract}
In order to reveal the present situation of teachers and students relationship, this paper conducted a survey on 266 university students. The results showed that: students who get an score in obstacles type dimension is equal or greater than 3 take a big proportion; for student whose score of two positive dimension is equal or greater than 3 account for over $50 \%$; there is gender difference in conflict type dimension $(\mathbf{p}<0.01)$; there is significant grade difference in conflict type dimension, barrier type dimension and cohesive type dimension $(p<0.01)$. Above conclusion suggests that it is necessary to study the relationship between teachers and students. Teachers and students should learn to communicate in network, and in that way to improve the relationship.
\end{abstract}

Keywords - university students, relationship, conflict type, barrier type, network communication platform

\section{高校师生关系的现状及基于网络的对策研究}

\author{
袁桂平 \\ 临沂大学教育学院, 临沂, 山东, 中国
}

摘 要 本研究采用问卷调查辅助工作, 对在校 266 名大学生进行了调查, 结果发现: 师生关系总体分析结果是障碍型维度均分 大于等于 3 的人数所占比例较大, 两个正向维度均分大于等于 3 的人数所占比例均大于 $50 \%$ 。大学师生关系在冲突型维度上存在性别 差异 $(p<0.01)$, 在冲突型、障碍型和亲密型三个维度上均存在显著的年级差异, 尤其是在亲密型维度上大四与其他三个年级存在显著 差异 ( $p<0.01)$ 。因此, 对高校师生关系进行研究是有必要的, 我们要学会利用网络来增加师生之间的交流, 从而使师生关系得到改善。

关键词 大学生, 师生关系, 冲突型, 障碍型, 网络交流平台

\section{1. 引言}

高校和谐师生关系的建构是和谐社会对高等学校教育 中师生关系提出的必然要求, 也是高等学校提高其教育教 学质量的重要保证。近年来, 随着我国经济的高速发展, 高校的不断扩招, 教师队伍的层次多样, 教师的师德、学 生的素质参差不齐, 高校教育管理方式相对陈旧和滞后等 问题, 严重影响了目前高校教育教学工作。在这样的环境 中, 高校师生沟通较少, 感情淡薄, 甚至有些学生与教师 沟通存在障碍。面对这种状况, 我们应该从教师和学生的 自身出发, 寻找相应的对策来改善师生关系。在当下的网 络时代, 我们应该充分利用网络资源, 充分利用网络交流 平台增加与老师的沟通交流, 从而改善师生关系。

\section{2. 问题提出}

教师是学生人际交往的重要对象, 师生关系是学生人 际交往中的重要内容, 对教育效果及学生成长有着重要影 响。在目前网络时代的背景下, 大学的师生关系是不容乐 观的, 学生与教师之间的沟通很少, 几乎是学生没有事不 去与教师沟通交流, 教师对学生的了解甚少, 因此构建和 谐师生关系是刻不容缓的。

\section{3. 研究程序}

\section{1 研究工具}

本研究对 Piana 的师生关系量表进行了修订, 采用修 订后的问卷进行调查。该问卷包含了 17 个项目四个维度 
(正向维度: 支持型和亲密型; 负向维度: 冲突型和障碍 型), 四个维度的信度均大于 0.75 , 量表采用的是五点计分 (从“很不符合”到“完全符合”)。被试在支持型和亲密型两个 维度上的得分越高, 表明师生关系越趋向于正向; 在冲突 型和障碍型两个维度上的得分越高, 则表明师生关系越趋 向于负向。

\section{2 研究对象}

本研究采用整群随机抽样法选取临沂大学 300 名在校 大学生, 发放问卷 300 份, 回收问卷 288 份, 有效问卷 266 份。其中男生 106 人，占总被试人数的 $39.8 \%$; 女生 160 人, 占总被试人数的 $60.2 \%$; 独生子女 98 人, 占总被试人 数的 $36.8 \%$; 非独生子女 168 人，占总被试人数的 $63.2 \%$;
大一学生 46 人, 占被试人数的 $17.3 \%$; 大二学生 44 人, 占被试人数的 $16.5 \%$; 大三学生 78 人, 占被试人数的 $29.3 \%$; 大四学生 98 人, 占被试人数的 $36.8 \%$ 。

\section{4. 研究结果}

4.1 高校师生关系的总体分析

本研究采用的量表是五点计分 (1、很不符合; 2、不 太符合； 3、一般； 4、比较符合; 5、完全符合), 统计维 度均分大于等于 3 的人数及所占的百分百是因为若维度均 分大于等于 3 就可以说明被试在这个维度上有倾向, 所占 的比例可以说明一些问题。统计结果如表 1 所示:

表 1 师生关系总体分析

\begin{tabular}{|c|c|c|c|c|}
\hline \multirow{2}{*}{} & \multicolumn{4}{|c|}{ 维 度 } \\
\cline { 2 - 5 } & 冲突型 & 障碍型 & 支持型 & 亲密型 \\
\hline 维度均分>=3 的人数 & 39 & 93 & 187 & 146 \\
\hline 占总人数的百分比 $(\%)$ & 14.7 & 35 & 70.3 & 54.9 \\
\hline
\end{tabular}

表 2 男女大学生的师生关系状况比较

\begin{tabular}{|c|c|c|c|c|c|}
\hline \multirow{2}{*}{} & \multicolumn{5}{|c|}{ 性 别 } \\
\cline { 2 - 6 } & \multicolumn{3}{|c|}{ 女 } \\
\cline { 2 - 6 } & $M$ & $S D$ & $M$ & $S D$ & $t$ \\
\hline 冲突型 & 11.27 & 4.217 & 9.04 & 3.374 & $4.784 * * *$ \\
\hline 障碍型 & 10.84 & 3.141 & 10.39 & 2.600 & 1.261 \\
\hline 支持型 & 13.10 & 3.132 & 13.04 & 2.915 & .160 \\
\hline 亲密型 & 11.89 & 3.287 & 11.89 & 2.794 & -.019 \\
\hline
\end{tabular}

注: *表示 $p<.05, * *$ 表示 $p<.01, * * *$ 表示 $p<.001$, 下同。

表 3 不同年级大学生的师生关系状况比较

\begin{tabular}{|c|c|c|c|c|c|c|c|c|c|}
\hline & \multicolumn{8}{|c|}{ 年 级 } & \multirow[b]{3}{*}{$F$} \\
\hline & \multicolumn{2}{|c|}{ 大一 $(n=46)$} & \multicolumn{2}{|c|}{ 大二 $(n=44)$} & \multicolumn{2}{|c|}{ 大三 $(n=78)$} & \multicolumn{2}{|c|}{ 大四 $(n=98)$} & \\
\hline & $M$ & $S D$ & $M$ & $S D$ & $M$ & $S D$ & $M$ & $S D$ & \\
\hline 冲突型 & 8.22 & 2.60 & 8.91 & 2.87 & 10.03 & 4.07 & 11.11 & 4.24 & $7.56 * * *$ \\
\hline 障碍型 & 9.80 & 2.47 & 10.27 & 2.44 & 10.65 & 3.23 & 11.07 & 2.78 & 2.34 \\
\hline 支持型 & 13.76 & 2.93 & 12.98 & 2.51 & 13.06 & 3.56 & 12.79 & 2.72 & 1.12 \\
\hline 亲密型 & 12.80 & 2.52 & 12.30 & 2.90 & 12.28 & 3.80 & 10.97 & 2.65 & $5.50 * *$ \\
\hline
\end{tabular}

4.2 男女大学生的师生关系状况比较

对于不同性别的大学生进行独立样本 $\mathrm{T}$ 检验, 检验结 果如表 2 所示。
由表 2 可以看出, 在师生关系冲突型维度上, 男生和 女生存在显著差异, 且男生平均分要高于女生; 而在师生 关系的其他三个维度上, 男女大学生的差异不显著。这说 
明当前大学生师生关系中, 男生与老师的关系不够和谐。 表 3 还显示出两个负向维度 (冲突型和障碍型) 的平均分 与两个正向维度 (支持型和亲密型) 的平均分相近。

\section{3 不同年级大学生的师生关系状况比较}

大学生师生关系状况的年级差异, 如表 3 所示。

由表 3 可知, 不同年级大学生在师生关系的冲突型和 亲密型两个维度上的得分存在显著的差异, 而在师生关系 的障碍型和支持型两个维度上的差异不显著。进一步多重 比较发现: 不同年级大学生师生关系在冲突型维度上的的 差异主要表现为大一与大三和大四存在显著差异, 且大三 明显高于大一 $(p=0.010<0.05)$, 大四也显著高于大一 ( $\mathrm{p}=0.000<0.001)$ 。大二与大四在这个维度上也存在显著差 异, 且大四明显高于大二 $(\mathrm{p}=0.001<0.01)$ 。在障碍型维度 上大一与大四也存在显著的差异, 大四显著高于大一 $(p=0.013<0.05)$, 这说明大一与老师沟通存在障碍。在支 持型维度上四个年级不存在差异。不同年级在亲密型维度 上存在显著差异, 大四与大一、大二、大三三个年级均存 在差异 $(p<0.05)$, 大四平均分均明显低于大一、大二、大 三。

\section{5 讨论及对策}

\section{1 讨论分析}

\subsection{1 高校师生关系总体分析}

本研究首先对师生关系总体进行了分析, 统计各维度 的均分, 计算出平均分大于等于 3 的人数, 然后算出他们 在总体占的百分比。结果显示, 被试在师生关系冲突型维 度上占了 $14.7 \%$, 虽然比例不是很大, 但是还是能说明一 些问题的, 说明还是有一部分同学与教师存在冲突关系的。 在障碍型维度上, 均分大于等于 3 的人数占 $35 \%$, 这说明 有一些同学与教师存在沟通障碍, 有些同学甚至不敢和教 师接触, 与教师接触他们会觉得不自在, 说明师生关系存 在明显的不和谐。在两个正向维度上, 在支持型维度上占 的比例最大, 均大于 $50 \%$, 在亲密型维度上次之, 这说明 一些同学只是可以在教师那里得到支持和鼓励, 但是还没 有达到一种亲密状态, 因此师生关系仍有待改善。

\subsection{2 高校师生关系的性别差异分析}

本研究结果显示, 在师生关系冲突型维度上男生的平 均得分要高于女生, 且两者具有显著性差异, 这说明男生 与教师的关系更为不和谐, 有时还会有冲突。而在障碍型 维度上二者得分均相当, 说明对于与教师沟通交流男女没 有差异。在支持型和亲密型维度上男女生也没有显著差异。
那么男生冲突型维度得分高的原因又是什么呢? 这可能是 因为男生本身的攻击性要强于女生, 对于教师的教学方式 以及平时的奖惩方式, 他们如果觉得不妥当他们就会表示 出不满, 并且据统计现在大学里男教师要多于女教师, 男 生发生冲突的对象更多的是男教师, 因此对教师的不满可 能会引起反抗，这就会导致冲突发生。

\subsection{3 高校师生关系的年级差异分析}

本研究结果显示, 在师生关系冲突型维度上大二、大 三、大四均高于大一。我觉得有以下两种原因导致大一新 生与老师冲突较小: 一方面, 大一学生刚刚走进大学校园, 他们是高考的幸运儿, 在初中高中时也应该是老师的得意 门生, 他们与大学教师接触还不是很多, 他们可能还停留 在初中高中与老师沟通多的状态中, 因此他们与大学教师 之间的接触不多, 还没有真正建立大学里的师生关系; 另 一方面, 大一学生刚进入大学, 对大学的学习、生活还显 得不习惯和不适应, 他们在高中时老师要求要严格, 而在 大学, 上课的模式他们刚刚接触, 觉得有些自由, 与教师 接触很少，因此与教师冲突也很少产生。

研究结果还显示出了在障碍型这个维度上大一与大四 存在显著差异, 并且大一在此维度上的得分也不是很低, 据统计对于障碍型包括的项目, 学生选择 3 分以上所占的 比例也相当高, 这个结果表明对于大学生而言, 他们与教 师沟通接触存在明显的障碍, 他们有的会觉得与教师相处 很费劲, 还有的为自己与教师的关系不好而发愁, 这些问 题都能显示出他们在与教师接触时有困难, 这样他们就会 减少与教师的沟通交流。

从研究结果还能看出, 在亲密型维度上大四与大一、 大二、大三三个年级均有显著差异。且大四得分均低于其 他三个年级, 造成这样的原因可能是因为大二大三的专业 课较多, 与教师接触的机会也较多, 但是到了大四, 由于 毕业在即, 有的忙于考研究生, 有的忙于找工作, 而且大 四一年的课程相对很少, 与教师接触和交流的机会也大幅 度减少, 故他们就不再像低年级学生与教师那样有着较亲 密的关系。

\subsection{4 高校师生关系影响因素分析}

影响高校师生关系的因素很多, 综合前人的研究, 我 认为影响高校师生关系的原因主要有以下几个方面: 首先, 一些高校扩招, 这就会影响师生交流的次数。众所周知, 人际关系的疏密, 与人际交往的频率、人际交往数量有关。 当前高校扩招、办学规模日渐扩大, 学生人数日益增多, 许多学校都会实行合堂上课, 教师常常面对百人以上的学 生上课, 这种状况就会减少师生接触的机会, 增加交往的 
难度, 很多教师几乎不认识学生, 更何况交流, 自然使师 生关系趋于淡漠。

最后, 现在社会对教师的要求增加, 教师只要稍有不 慎就会招致各种各样的批评。在这种情况下, 学生不能以 平常的人性化的标准看待教师, 他们认为教师不应该有任 何错误, 既学富五车又品德高尚。因此, 教师稍有差错就 会令学生抱怨, 甚至容易因教师的一点失误或差错就以偏 概全, 完全否定教师, 产生失望情绪, 从而有意不与教师 深入交往, 影响师生关系。

\section{2 相关对策}

\subsection{1 网络环境下师生关系的重构}

教育过程中的知识传递、情感陶冶、人格培养等, 都 基于师生关系而形成。我们生活在网络纵横的年代, 十年 前, 网络还处于萌芽时期, 那时很少有学生拥有手机、电 脑。随着经济的发展, 网络的普及, 现如今在网络的环境 中我们应该充分利用网络资源来满足我们的日常需要。网 络的便捷性、交互性、超时空性以及对资源的共享分配方 式对缓和紧张的师生关系有着积极影响, 对构建新型的师 生关系也起着重要的作用。

\subsection{2 网络交流平台的应用}

在新的媒体中, 教师与学生的对话方式、交流方式、 交际平台的构建可以有多种方式, 其中主要有手机、电子 邮件、飞信、博客等方式。

\subsubsection{1 手机}

对于本研究中发现的问题, 男生与教师更容易发生冲 突, 这可能是男生很难理解教师, 也应该是与教师沟通较 少的结果, 我们可以利用手机来增加与教师的沟通交流, 使学生与教师相互了解, 相互理解, 这样就会减少矛盾和 冲突, 增加和谐。手机通话是一种非面对面的语言沟通。 通过运用手机通话可以增加师生之间的交流沟通, 在高校 师生沟通中有时会有某种原因不愿意把自己的一些意见明 确地表达出来, 这时, 就可以采用模糊的技巧把输出的信 息“模糊化”, 使师生之间的沟通留有余地, 这样就避免了 面对面交流的尴尬局面。我们也可以通过手机短信或者发 飞信的方式, 对于一些问题, 学生不想当面表达, 可以通 过短信来沟通。通过手机还可以从大学生那获得关于教学 方面的信息，从而及时、有效地改善师生间的关系。

\subsubsection{2 电子邮件}

本研究还发现, 大一与教师沟通存在明显的障碍。的 确如此, 在师生关系整体研究中发现师生关系有一定比例 的冲突型和障碍型, 且障碍型比例较高。他们可能是刚刚 进入大学, 与教师沟通很少, 甚至有的学生不知道该怎么
去和教师交流，不敢与教师交流。对于不敢表达的思想， 我们可以通过给教师发送电子邮件来表达, 它不仅方便快 捷而且隐蔽性很强。有些大学生与教师交流会紧张, 与教 师面对面交流可能不能完全表达他们的想法, 因此学生可 以运用电子邮件的形式把他们的一些心理的问题直接发给 教师, 让教师给他们帮助, 电子邮件不可替代的优点就是 可以传输大篇幅的内容, 并且隐蔽性很强, 这样就可以避 免让更多的人知道, 而且效果也会更加明显。这样可以一 点点的减少交流障碍, 从而加强支持、增进师生间的亲密 感。

\subsubsection{3 博客}

本研究显示出大四学生与教师的亲密性很低, 说明大 四学生与教师交流较少, 大四考研的同学时间很紧, 有了 博客, 他们可以随时抽出一点时间来看看这个交流平台上 的情况, 来看看有没有自己学习上需要的资料, 这样还可 以促进师生之间的交流。博客(Blog) 就是以网络作为载体, 简易迅速便捷地发布自己的心得, 及时有效轻松地与他人 进行交流, 再集丰富多彩的个性化展示于一体的综合性平 台, 博客具有半开放性的特点。博客可以讨论某个议题或 发表一些言论, 教师可以将自己的信息发送的博客网站, 这是汇报自己的信息而不是直接发给接受者, 接受者 (学 生) 可以反复阅读, 这样学生就可以得到信息的真实内涵, 从而进行周密思考。

除了这几个网络沟通工具之外还有很多种方式进行网 上交流，我们应该充分利用现在的网络资源，我相信网络 在给我们带来方便的同时还可以增进师生之间的交流，拉 近师生之间的距离。

\section{参考文献(References)}

[1] Shilong Fan. The Study on Improve Strategies of the Harmonious Relationship between students and teachers. Northeast Normal University,2007.

[2] Mitchell.The Relationship Between Teacher Behaviours and Student Talkin Promoting Quality Learning in Science Classrooms.Research in Science Education,2010.40(2):p.171 -186 .

[3] Weiming Peng. Alteration and orientation of university teacher-student. Journal of Higher Education,2002.23(5):p.7578.

[4] Yuanli Li.Study on Constructing Harmonious Relationship between University. Jiangxi Normal University,2007.

[5] Huichun yu.The Problems, Reflection and Restructuring of Relationship Between Teacher and Student in University. Journal of Yunmeng,2008.29(6):p.110-112. 